\title{
Voronoi Cell Geometry Based Dynamic Fractional Frequency Reuse for OFDMA Cellular Networks
}

\author{
Rahat Ullah, Norsheila Fisal, Hashim Safdar, Wajahat Maqbool, Zubair Khalid, A.S. Khan \\ UTM-MIMOS, Center of Excellence for Telecommunication Technology. \\ Faculty of Electrical Engineering, Universiti Teknologi Malaysia (UTM) \\ Skudai, 81310, Johor, Malaysia \\ eng.rahat.u@ieee.org, sheila@fke.utm.my,\{eng.hashim.s,eng.wajahat.m\}@ieee.org, \\ \{engr.zktanoli, adnan.ucit\}@gmail.com
}

\begin{abstract}
Interference Management (IM) is one of the major challenges of next generation wireless communication. Fractional Frequency Reuse (FFR) has been acknowledged as an efficient IM technique, which offers significant capacity enhancement and improve cell edge coverage with low complexity. In literature, FFR has been analyzed mostly with cellular networks described by Hexagon Grid Model, which is neither tractable nor scalable to the dense deployment of next generation wireless networks. Moreover, the perfect geometry based grid model tends to overestimate the system performance and not able to reflect the reality. In this paper, we use the stochastic geometry approach, FFR is analyzed with cellular network modeled by homogeneous Poisson Point Process (PPP). A dynamic frequency allocation scheme is proposed which take into account the randomness of the cell coverage area describe by Voronoi tessellation. It is shown that the proposed scheme outperforms the traditional fixed frequency allocation schemes in terms of per user capacity and capacity density.
\end{abstract}

Index Terms-Inter-Cell Interference (ICI), Interference Management, Fractional Frequency Reuse, Long Term Evolution (LTE), Poisson Point Process

\section{INTRODUCTION}

Recent trends in the bandwidth hungry applications has resulted in a drastic growth of the data traffic, forcing network operators to significantly increase their system capacity. Modern cellular systems are changing rapidly and are more crowded in terms of base stations deployment. This trend has triggered the development of new cellular standards, of which LTE and LTE-Advaced by Third Generation Partnership Project (3GPP) are the more promising candidates. To accomplish high throughput and enhanced spectral efficiency, LTE is based on Orthogonal Frequency Division Multiple Access (OFDMA). Because of OFDMA, Intra-cell interference is no more an issue due to orthogonal carriers for each users in the same cell, however, ICI is the primary source of interference and one of the prominent limiting factor in performance degradation. ICI problem is more severe especially at the cell edges.

To enhance the performance of the cellular network, Intercell Interference Coordination (ICIC) is an approach [1] [2], where each cell is allocating its resources in a way such that to minimize the overall interference experienced in the network and to maximize the spatial reuse. Fractional frequency reuse
(FFR) has been anticipated as an attractive ICIC technique in OFDMA based wireless cellular networks due to its low complexity and its offered higher spectral efficiency [3]. FFR is the part of fourth generation (4G) standards since Release 8 [2]. The main objective of FFR is to improve the SINR and system throughput by reducing Co-Channel Interference (CCI) and ICI.

The rest of the paper is organized as in section II interference management with FFR is explained. Network topology and related work is presented in section III. Section IV gives model description and proposed scheme. Results and discussions are given at section $\mathrm{V}$, whereas conclusion is presented in section VI .

\section{INTERFERENCE MANAGEMENT WITH FFR}

FFR aims to catch the middle ground between the full frequency reuse at the cost of high interference and partial frequency reuse which avoid the interference by sharing the frequency resources orthogonality among the neighbors cells. From an interference point of view, all users within a cell are not suppose to be the same because of the path loss effect and different SINR values. Users located at the cell edges are more sensitive to interference compared to the users at the cell center. FFR combines the benefits of both low and high frequency reuse factor by dividing the users within the cell area into two regions. (i) Cell center region where users are close to the base station (ii) Cell edge region where the users are more suited to the border of the cell. The inception behind FFR is basically the partition and allocation of cell's bandwidth in such a way that the interference is avoided in adjacent cell for edge users [4]. Whereas, interference received or created by the cell center users is greatly reduced. Moreover, FFR offers higher spectral efficiency as compared to conventional frequency reuse [2]-[6].

In LTE with OFMDA, 12 subcarriers of $15 \mathrm{KHz}$ each are grouped into a physical Resource Block (RB). Each RB is equal to $180 \mathrm{KHz}$ of spectrum can be allocated with different options according to FFR scheme which essentially based on allocating a number of these RBs in a sector. As an interference avoidance scheme, FFR ensures to avoid collision of same frequency band at the adjacent cells by allocating different frequencies either in a static way or by scheduling 